\title{
HOLOMORPHIC IMMERSIONS OF A COMPACT KÄHLER MANIFOLD INTO COMPLEX TORI
}

\author{
YOZO MATSUSHIMA
}

In this paper we shall study the holomorphic immersion of a compact connected Kähler manifold $M$ into a complex torus. It is easily seen that $M$ admits a holomorphic immersion into a cmoplex torus if and only if the holomorphic cotangent bundle $T^{*}(M)$ is ample (see $\S 1$ ), and it has been proved in a joint paper [6] by the author with W. Stoll that if $T^{*}(M)$ is ample and one of the Chern numbers of $M$ is nonzero, then $M$ is algebraic.

This paper is devoted mainly to the study of a compact connected $n$-dimensional Kähler manifold $M$ admitting a holomorphic immersion $\Phi$ into an $(n+1)$-dimensional complex torus $B$. The image $X=\Phi(M)$ defines a positive irreducible divisor $D(X)$ and a holomorphic line bundle which we shall denote by $\{X\}$. The Chern class $c(\{X\})^{\text {o }}$ of $\{X\}$ is represented by a unique $(1,1)-$ form of the type $\frac{1}{2} i \sum h_{k j} \zeta^{k} \wedge \bar{\zeta}^{j}$, where $\left\{\zeta^{1}, \cdots, \zeta^{n+1}\right\}$ is a basis of the space of holomorphic 1 -forms on $B$, and $H_{\Phi}=\left(h_{k j}\right)$ is a constant Hermitian matrix. It is known from the theory of theta functions that the Hermitian matrix $H_{\Phi}$ is positive (Weil [9]). Our main purpose is to describe the properties of $M$ in term of the Hermitian form $H_{\Phi}$, and our main results are as follows.

First we show that we can reduce the case, where $H_{\Phi}$ is degenerate, to the case where $H_{\Phi}$ is positive definite. Namely let $\operatorname{Aut}_{0}(M)$ be the identity component of the group of holomorphic transformations of $M$. It is well-known that the group $\operatorname{Aut}_{0}(M)$ is a complex Lie group, and in our special case we prove that $\operatorname{Aut}_{0}(M)$ is a complex torus acting freely on $M$ and the complex dimension of $\mathrm{Aut}_{0}(M)$ is equal to the nullity of the Hermitian form $H_{\Phi}$. Then $M$ is a holomorphic principal bundle over the quotient $N=M / \operatorname{Aut}_{0}(M)$ with structure group $\operatorname{Aut}_{0}(M)$. The quotient manifold $N$ is algebraic and admits a holomorphic immersion $\Psi$ into a complex torus whose complex dimension is $\operatorname{dim} N+1$, and the Hermitian matrix $H_{\Psi}$ associated with the immersion $\Psi$ is positive definite. We shall show that the following conditions are equivalent: 1) $H_{\phi}$ is positive definite, 2) $\left.\operatorname{dim} \operatorname{Aut}_{0}(M)=0,3\right)$ the Euler number $E(M)$ of $M$ is nonzero.

We then prove that a compact connected $n$-dimensional Kähler manifold $M$ with $E(M) \neq 0$ admits a holomorphic immersion into an $(n+1)$-dimensional complex torus if and only if the holomorphic cotangent bundle of $M$

Communicated May 9, 1973. 
is ample and $M$ has precisely $n+1$ linearly independent holomorphic 1 -forms. Furthermore, the Albanese variety $A$ of $M$ is $(n+1)$-dimensional, the canonical map $J: M \rightarrow A$ is an immersion, and any holomorphic immersion $\Phi$ of $M$ into an $(n+1)$-dimensional complex torus $B$ is obtained from the canonical map $J$ composing with a homomorphism of $A$ onto $B$ and a translation of $B$.

It is shown that every divisor in $M$ is a divisor of a "theta function" on the universal covering manifold of $M$, and we shall obtain expressions of the Euler number, the arithmetic genus and the plurigenera of $M$ in terms of the elementary divisors of the imaginary part of the Hermitian matrix $H_{J}$ associated with the canonical map $J: M \rightarrow A$ and the "degree" of $J$.

The author wishes to thank Professors T. Nagano and B. Smyth who showed the author their recent results on the minimal immersions of a compact manifold into real tori. The presentation of $\S 1$ of this paper is greatly influenced by a conversation with them.

1. Let $B=C^{m} / \Delta$ be a complex torus of dimension $m$, where $\Delta$ is a lattice in $\boldsymbol{C}^{m}$. We shall denote by $\pi$ the canonical projection of $\boldsymbol{C}^{m}$ onto $\boldsymbol{B}$. Let $\left\{\boldsymbol{w}^{1}, \cdots, \boldsymbol{w}^{m}\right\}$ be the standard coordinates in $\boldsymbol{C}^{m}$. Then 1 -forms $d w^{k}$ are invariant by translations and hence projectable onto $B$. There exist therefore $m$ linearly independent holomorphic 1 -forms $\zeta^{1}, \ldots, \zeta^{m}$ on $B$ such that

$$
\pi^{*} \zeta^{k}=d w^{k} \quad(k=1, \cdots, m)
$$

and these 1-forms are invariant by translations in $B$ and form a basis of all holomorphic 1-forms in $B$. We shall denote by $T_{b}$ the translation of $B$ by an element $b \in B$. We identify the holomorphic tangent vector space $T_{y}(B)$ of $B$ at each point $y \in B$ with $C^{m}$ by the identification map $T_{y}(B) \rightarrow C^{m}$ which assigns to each vector $u \in T_{y}(B)$ the $m$-dimensional vector $\left(\zeta^{1}(u), \cdots, \zeta^{m}(u)\right) \epsilon$ $C^{m}$.

Let $M$ be an $n$-dimensional complex manifold, and

$$
\Phi: M \rightarrow B
$$

be a holomorphic map from $M$ into $B$, and let

$$
\omega^{k}=\Phi^{*} \zeta^{k} \quad(k=1, \cdots, m) .
$$

Then $\omega^{1}, \cdots, \omega^{m}$ are holomorphic 1 -forms on $M$ and the differential $\Phi_{*}(x)$ : $T_{x}(M) \rightarrow T_{\Phi(y)}(B)=C^{m}$ is given by

$$
\Phi_{*}(x)(u)=\left(\omega^{1}(u), \cdots, \omega^{m}(u)\right), \quad u \in T_{x}(M) .
$$

Let $x_{0}$ be the reference point of $M$ chosen once for all. Then

$$
\Phi(x)=T_{\Phi\left(x_{0}\right)}\left(\pi\left(\int_{x_{0}}^{x} \omega^{1}, \cdots, \int_{x_{0}}^{x} \omega^{m}\right)\right),
$$


where $\int_{x_{0}}^{x} \omega^{k}$ means the integral of $\omega^{k}$ along a path $\gamma$ from $x_{0}$ to $x$, and it can be shown that $\pi\left(\int_{x_{0}}^{x} \omega^{1}, \cdots, \int_{x_{0}}^{x} \omega^{m}\right)$ does not depend on the choice of $\gamma$.

Let $\Phi_{1}$ and $\Phi_{2}$ be two homotopic holomorphic maps from $M$ to $B$, and assume that $M$ is compact. Since $\Phi_{1}$ and $\Phi_{2}$ are homotopic, closed holomorphic forms $\Phi_{1}^{*} \zeta^{k}$ and $\Phi_{2}^{*} \zeta^{k}$ have the same periods and are therefore cohomologous. Thus $\Phi_{1}^{*} \zeta^{k}-\Phi_{2}^{*} \zeta^{k}=d f$ and $d f=d^{\prime} f, d^{\prime \prime} f=0$, so that $f$ is holomorphic and hence a constant, and we get $\Phi_{1}^{*} \zeta^{k}=\Phi_{2}^{*} \zeta^{k}$. It follows then from (1.3) that

$$
\Phi_{2}(x)=T_{b}\left(\Phi_{1}(x)\right), \quad b=\Phi_{2}\left(x_{0}\right) \cdot \Phi_{1}\left(x_{0}\right)^{-1} .
$$

From now on we always assume that $M$ is compact and connected, and let Aut $(M)$ be the group of all holomorphic transformations of $M$. It is wellknown that Aut $(M)$ has a structure of complex Lie group such that Aut $(M) \times$ $M \rightarrow M$ defined by $(g, x) \rightarrow g \cdot x$ is a holomorphic map. We shall denote by $\operatorname{Aut}_{0}(M)$ the identity component of Aut $(M)$. If $g \in \mathrm{Aut}_{0}(M)$, then $g$ is homotopic to the identity map of $M$, and hence $\Phi$ and $\Phi \circ g$ are homotopic for any holomorphic map $\Phi$ from $M$ to $B$. Therefore

$$
\Phi(g x)=T_{\Phi^{\prime}(g)}(\Phi(x)),
$$

where

$$
\Phi^{\prime}(g)=\Phi\left(g x_{0}\right) \circ \Phi\left(x_{0}\right)^{-1} .
$$

It is easily seen that $\Phi^{\prime}$ is a homomorphism of the complex Lie group Aut $(M)$ into the complex torus $B$. The Lie algebra \&S of all holomorphic vector fields on $M$ is identified with the Lie algebra of $\operatorname{Aut}_{0}(M)$. The Lie algebra of $B$ is also identified with the Lie algebra of all translation invariant vector fields $X$ of type $(1,0)$ which is identified with $C^{m}$ by the map $X \rightarrow\left(\zeta^{1}(X), \cdots, \zeta^{m}(X)\right)$. Then the homomorphism of Lie algebra $\Phi_{*}^{\prime}:(S) \rightarrow C^{m}$ induced by $\Phi^{\prime}:$ Aut $_{0}$ $(M) \rightarrow B$ is given by

$$
\Phi_{*}^{\prime}(\xi)=\left(\omega^{1}(\xi), \cdots, \omega^{n}(\xi)\right),
$$

where $\xi \in \mathbb{S}$ is a holomorphic vector field on $M$.

Let $M$ be a compact connected Kähler manifold, $\left\{\theta^{1}, \cdots, \theta^{q}\right\}\left(q=h^{1,0}(M)\right)$ a basis of the vector space of all holomorphic 1-forms on $M$, and $H_{1}(M, Z)$ the 1-dimensional integral cohomology group of $M$. Then the image $\Delta_{A}$ of the homomorphism $\gamma \rightarrow\left(\int_{\gamma} \theta^{1}, \cdots, \int_{\gamma} \theta^{q}\right)$ of $H_{1}(M, Z)$ into $C^{q}$ is a lattice of $C^{q}$, and the complex torus

$$
A=C^{q} / \Delta_{A}
$$

is called the Albanese variety of $M$. The map $J: M \rightarrow A$ defined by 


$$
J(x)=\pi\left(\int_{x_{0}}^{x} \theta^{1}, \cdots, \int_{x_{0}}^{x} \theta^{q}\right)
$$

is holomorphic, and $J^{*}\left(\zeta^{k}\right)=\theta^{k}(k=1, \cdots, q)$. We call $J$ the canonical map of $M$ into $A$.

If $\Phi: \rightarrow B$ is a holomorphic map of $M$ into a complex torus $B$, then there exists a homomorphism $f: A \rightarrow B$ such that

$$
\Phi=T_{\Phi\left(x_{0}\right)} \circ f \circ J
$$

Assume now that $M$ is compact and connected, and let us denote by $\mathfrak{h}$ the complex vector space of all holomorphic 1-forms on $M$. We may of course identify $\mathfrak{h}$ with the vector space $\Gamma\left(T^{*}(M)\right)$ of holomorphic sections of the holomorphic cotangent bundle $T^{*}(M)$. Let $e_{x}(x \in M)$ be the linear map $\mathfrak{h} \rightarrow$ $T_{x}^{*}(M)$ defined by $\omega \rightarrow \omega(x)$ for $\omega \in \mathfrak{h}$. We say that $T^{*}(M)$ is ample if $e_{x}$ is surjective for all $x \in M$.

Assume now that there exists a holomorphic map $\Phi$ of $M$ into a complex torus $B$, and let $V$ be the subspace of $\mathfrak{h}$ spanned by $\omega^{1}, \cdots, \omega^{m}$, where $\omega^{k}$ are defined by (1.1). By (1.2) the differential $\Phi_{*}(x)$ of $\Phi_{*}$ at $x$ is injective if and only if $e_{x} V=T_{x}^{*}(M)$. Therefore, if $\Phi$ is an immersion, then $e_{x} V=T_{x}^{*}(M)$ for every $x$. This shows that if $M$ admits a holomorphic immersion into a complex torus, then $T^{*}(M)$ is ample. Conversely, if $T^{*}(M)$ is ample and $M$ is Kählerian, then the canonical map $J$ is a holomorphic immersion of $M$ into the Albanese variety $A$.

Lemma 1. Let $M$ be a compact connected $n$-dimensional complex manifold. Assume that $M$ admits a holomorphic immersion $\Phi$ into a complex torus $B$. Then the group $\mathrm{Aut}_{0}(M)$ is a complex torus and acts freely on M. Moreover, the kernel of the homomorphism $\Phi^{\prime}: \operatorname{Aut}_{0}(M) \rightarrow B$ is finite.

Proof. The homomorphism $\Phi_{*}^{\prime}: \mathbb{B} \rightarrow C^{m}$ is injective. In fact, if $\xi \in \mathbb{S}$ and $\Phi_{*}^{\prime}(\xi)=0$, then $\omega^{k}(\xi)=0(k=1, \cdots, m)$ by $(1.4)$, so that $\xi=0$ because $\Phi$ is an immersion. Let $\xi \in$ (S), $\xi \neq 0$, and $\Phi_{*}^{\prime}(\xi)=a$, and let $h_{t}$ be the 1-parameter group of holomorphic transformations of $M$ generated by $\xi$. Then $\Phi\left(h_{t}\right)$ $=T_{\pi(\mathrm{ta})}$ for all $t \in R$, and the 1-parameter group of translations $T_{\pi \text { (ta) }}$ leaves the image $\Phi(M)$ invariant. Let $g_{0}=\zeta^{1 \bar{\zeta}^{1}}+\cdots+\zeta^{m} \bar{\zeta}^{m}$ be the flat Kähler metric in the torus $B$, and $g=\Phi^{*} g_{0}$ the pullback of $g_{0}$ by $\Phi$. Then $g$ is a Kähler metric of $M$. We show that $h_{t}$ is an isometry for all $t \in R$. Let $y_{0}$ be an arbitrary point of $M$, and $U$ a neighborhood of $y_{0}$ such that $\Phi$ maps $U$ biholomorphically onto a submanifold $\Phi(U)$ of $B$. Then $a=\Phi_{*}^{\prime}(\xi)$ is tangent to $\Phi(U)$. Moreover, $T_{\pi \text { (ta) }}$ is an isometry of $B$ (with respect to $g_{0}$ ) for each $t$, and leaves $\Phi(U)$ invariant provided $|t|$ is sufficiently small. It follows that $a$ is an infinitesimal isometry (Killing vector field) of $\Phi(U)$, and therefore that the restriction of $\xi$ to $U$ is also an infinitesimal isometry in $U$ with respect to $g$. Thus $\xi$ is an infinitesimal isometry of the Kähler manifold $M$, and $h_{t}$ is an isometry of $M$ for each $t$. Since it is known that every infinitesimal isometry of a compact 
Kähler manifold is a holomorphic vector field (cf. [2]), the group $\mathrm{Aut}_{0}(M)$ coincides with the identity component of the group of all isometries of $M$ and is therefore compact. Since the kernel of $\Phi_{*}^{\prime}: \mathbb{S} \rightarrow C^{m}$ is trivial and Aut $_{0}(M)$ is compact, the kernel of the homomorphism $\Phi^{\prime}: \operatorname{Aut}_{0}(M) \rightarrow B$ is finite. Now let $g \in \operatorname{Aut}_{0}(M)$ and $g y_{0}=y_{0}$ for some $y_{0} \in M$. Then $\Phi\left(y_{0}\right)=\Phi^{\prime}(g) \Phi\left(y_{0}\right)$ and $\Phi^{\prime}(g)=e$, the identity element of $B$. Hence $\Phi(g x)=\Phi(x)$ for any $x \in M$, and $\Phi_{*}\left(x_{0}\right) \circ g_{*}\left(x_{0}\right)=\Phi_{*}\left(x_{0}\right)$, where $g_{*}\left(x_{0}\right)$ denotes the differential of $g$ at $x_{0}$. Since $\Phi_{*}\left(x_{0}\right)$ is injective, $g_{*}\left(x_{0}\right)$ should be the identity map. On the other hand, $g$ is an isometry of $M$ and maps a geodesic $\sigma$ starting at $x_{0}$ with direction $u$ to a geodesic $g \cdot \sigma$ starting at $g x_{0}$ with direction $g_{*}\left(x_{0}\right) u$. Since $g\left(x_{0}\right)=x_{0}$ and $g_{*}\left(x_{0}\right) u$ $=u$ for any $u, g$ leaves invariant any such geodesic pointwise. It follows then that $g$ is the identity map in a neighborhood of $x_{0}$. Since this holds for any fixed point $x_{0}$ of $g$, we can conclude that $g$ is the identity map, so that Aut $_{0}$ $(M)$ acts freely on $M$. Since $\operatorname{Aut}_{0}(M)$ is complex and compact, it is a complex torus.

Lemma 2. Under the assumption in Lemma 1, let $\Gamma$ be the closed subgroup of $B$ consisting of all $b \in B$ such that $T_{b} X=X$, where $X=\Phi(M)$ is the image of $M$. Then the identity component $\Gamma_{0}$ of $\Gamma$ coincides with the image $C=\Phi^{\prime}\left(\operatorname{Aut}_{0}(M)\right)$ of $\mathrm{Aut}_{0}(M)$.

Proof. Clearly $\Gamma$ is a closed subgroup of $B$ and hence a closed Lie subgroup of $B$. Let $b_{t}$ a 1-parameter subgroup of $\Gamma$. Then there exists a vector $a \in C^{m}$ such that $b_{t}=\pi(\mathrm{ta})$. Let $y_{0} \in M$, and let $U$ be a neighborhood of $y_{0}$ such that $\Phi$ maps $U$ biholomorphically onto a submanifold $\Phi(U)$ of $B$. As in the proof of Lemma 1, we see that $a$ is tangent to $\Phi(U)$ and is an infinitesimal isometry of $\Phi(U)$ with respect the Kähler metric of $\Phi(U)$ induced by the flat Kähler metric $g_{0}$ of $B$. Thus the vector field $\xi_{U}$ on $U$ corresponding to the restriction of $a$ to $\Phi(U)$ is an infinitesimal isometry of $M$ defined on $U$. Now let $a_{k}=\xi^{k}(a)$ and $\theta_{a}=\sum_{k=1}^{m} a_{k} \xi^{k}$. Then $\theta_{a}$ is a $(0,1)$-form on $B$ such that $\theta_{a}(\bar{v})$ $=g_{0}(a, \bar{v})$ for any $v \in C^{m}$, so that $\Phi^{*} \theta_{a}$ is a $(0,1)$-form on $M$ and there exists a unique vector field $\xi$ of type $(1,0)$ such that $g(\xi, \bar{\eta})=\left(\Phi^{*} \theta_{a}\right)(\bar{\eta})$ for any vector field $\eta$ of type $(1,0)$ on $M$. We show that $\xi=\xi_{U}$ on $U$. Let $x \in U$. Then $\Phi_{*}(x) \xi_{U}=a$ and $g\left(\xi_{U}(x), \bar{\eta}(x)\right)=g_{0}\left(a, \Phi_{*}(x) \bar{\eta}(x)\right)=\theta_{a}\left(\Phi_{*}(x) \bar{\eta}(x)\right)=$ $\left(\Phi^{*} \theta_{a}\right)(\bar{\eta}(x))$. Therefore $\xi(x)=\xi_{U}(x)$ and $\xi=\xi_{U}$ on $U$, which implies that $\xi$ is an infinitesimal isometry on $U$. Since $U$ is a neighborhood of an arbitrarily chosen point $y_{0} \in M, \xi$ is an infinitesimal isometry on $M$ and hence holomorphic. Moreover, $\Phi_{*}(x) \xi(x)=a$ at each point as the above proof shows. Then $\Phi^{\prime}(\xi)=a$, from which it follows that the one-parameter subgroup $b_{t}=\pi(\mathrm{ta})$ is contained in $C=\Phi^{\prime}\left(\operatorname{Aut}_{0}(M)\right)$ so that $L_{0} \subset C$. Since clearly $C \subset \Gamma_{0}$, $\Gamma_{0}=C$. q.e.d.

Since $\operatorname{Aut}_{0}(M)$ acts freely on $M$, the quotient $N=M / \operatorname{Aut}_{0}(M)$ is also a compact connected complex manifold. The image $C=\Phi^{\prime}\left(\mathrm{Aut}_{0}(M)\right)$ in $B$ is a compact subgroup of $B$ and moreover, since $\Phi^{\prime}$ is a complex Lie group homomorphism, $C$ is a complex subgroup and hence $C$ is a complex subtorus of $B$. 
The map $\Phi$ induces a holomorphic map $\Psi$ of $N$ into $B^{\prime}=B / C$ such that the diagram

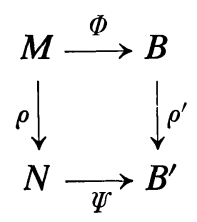

is commutative, where $\rho$ and $\rho^{\prime}$ denote the canonical projection of $M$ and $B$ onto $N$ and $B^{\prime}$ respectively.

It is easily seen that $\Psi$ is a holomorphic immersion of $N$ into $B^{\prime}$. We show that the group $\operatorname{Aut}_{0}(N)$ consists of only the identity element. To see this let $\Gamma_{0}^{\prime}$ be the identity component of the group of all $b^{\prime} \in B^{\prime}$ such that $T_{b}, X^{\prime}=X^{\prime}$, where $X^{\prime}=\Psi(N)$. Let $b^{\prime}(t)$ be a path in $\Gamma_{0}^{\prime}$ such that $b^{\prime}(0)=e^{\prime}, e^{\prime}$ being the identity element of $B^{\prime}$, and let $b(t)$ be a path in $B$ such that $\rho^{\prime}(b(t))=b^{\prime}(t)$ and $b(0)=e$. Let $x$ be an arbitrary point of $M$, and let $x^{\prime}=\rho(x)$. Then $T_{b^{\prime}(t)} \Psi\left(x^{\prime}\right) \in X^{\prime}$, and hence there exists an element $y^{\prime} \in N$ such that $T_{b^{\prime}(t)} \Psi\left(x^{\prime}\right)$ $=\Psi\left(y^{\prime}\right)$, where the parameter $t$ is fixed. Let $y$ be an element of $M$ such that $\rho(x)=y^{\prime}$. Then $T_{\rho^{\prime}(b(t))} \Psi(\rho(x))=\Psi(\rho(y))$, and from the commutativity of the diagram (1.5) we get $\rho^{\prime}(\Phi(y))=\rho^{\prime}\left(T_{b(t)} \Phi(x)\right)$. Thus there exists an element $c \in C$ such that $\Phi(y)=T_{c}\left(T_{b(t)} \Phi(x)\right)=T_{c b(t)} \Phi(x)$. This shows that $T_{c b(t)} X=$ $X$, where $X=\Phi(M)$, which implies that $T_{b(t)} X=T_{c^{-1}} X=X$, so that $b(t) \in$ $\Gamma$ in the notation of Lemma 2 for each $t$ and hence that $b(t) \in \Gamma_{0}=C$. Then $\rho^{\prime}(b(t))=e^{\prime}$, and $b^{\prime}(t)=e^{\prime}$ for all $t$, which proves that $\Gamma_{0}^{\prime}$ reduces to the identity element. Thus by Lemma 2 (applied for $N$ and $\Psi$ ) we see that Aut $_{0}$ $(N)$ is trivial, and hence the following proposition.

Proposition 1. Let $M$ be a compact connected complex manifold, and assume that $M$ admits a holomorphic immersion $\Phi$ into a complex torus $B$. Let Aut $_{0}(M)$ be the identity component of the complex Lie group of all holomorphic transformations of $M$. Then $\mathrm{Aut}_{0}(M)$ is a complex torus acting freely on $M$. Let $N=M /$ Aut $_{0}(M)$ be the quotient of $M$ by the free action of Aut $_{0}$ (M). Then $N$ is a compact connected complex manifold, $N$ admits also a holomorphic immersion in a complex torus, $\operatorname{Aut}_{0}(N)$ is trivial, and the manifold $M$ is a holomorphic principal fibre bundle over $N$ of the structure group Aut $_{0}$ (M).

2. In the following sections we always denote by $M$ a compact connected complex $n$-dimensional manifold, and assume that $M$ admits a holomorphic immersion $\Phi$ into an $(n+1)$-dimensional complex torus $B$.

Let $X=\Phi(M)$. Then $X$ defines a positive irreducible divisor $D(X)$ of $B$. More precisely there are an open covering $\left\{U_{\alpha}\right\}_{\alpha \in A}$ of $B$ and holomorphic functions $\left\{f_{\alpha}\right\}_{\alpha \in A}$, each $f_{\alpha}$ being defined on $U_{\alpha}$, such that $f_{\alpha} / f_{\beta}$ is holomorphic and nonvanishing on the intersection $U_{\alpha} \cap U_{\beta}$ and $X \cap U_{\alpha}$ is defined by the equa- 
tion $f_{\alpha}=0$ for each $\alpha$. We can define $\left\{f_{\alpha}\right\}$ in the following way. Let $p \in B$. If $p \notin X$, then choose a neighborhood $U(p)$ of $p$ such that $U(p) \cap X=\emptyset$, and define $f^{(p)} \equiv 1$. If $p \in X$, each preimage of $p$ in $M$ has a neighborhood which is mapped biholomorphically onto an $n$-dimensional submanifold of $B$ passing through $p$, and we obtain a finite number of distinct $n$-dimensional submanifolds $X_{1}, \cdots, X_{k}$ each of which passes through $p$ and is defined in a neighborhood $U(p)$ of $p$ by an equation $f_{j}=0$ such that $\left(d f_{j}\right)(p) \neq 0$, where $f_{j}$ is holomorphic in $U(p)$. Then define $f^{(p)}=f_{1}, \cdots, f_{k}$. The simple point of $X$ is the point $p \in X$ for which $k=1$. It is not difficult to check that the open covering $\{U(p)\}_{p \in B}$ and the holomorphic functions $\left\{f^{(p)}\right\}_{p \in B}$ verify the properties mentioned above, the set of simple points of $X$ is a connected $n$-dimentional submanifold of $B$, and, for a simple point $p, X$ is defined in a neighborhood of $p$ by a single equation $f=0$ such that $(d f)(p) \neq 0$. This means that the positive divisor $D(X)$ is irreducible (cf. Weil [9, Appendix]). In the above notation put $g_{\alpha \beta}=f_{\alpha} / f_{\beta}$. Then $g_{\alpha \beta} \cdot g_{\beta \gamma}=g_{\alpha \gamma}$ on $U_{\alpha} \cap U_{\beta} \cap U_{\gamma}$, and $\left\{g_{\alpha \beta}\right\}$ is a system of transition functions of a holomorphic line bundle which we shall denote by $\{X\}$.

We recall here several facts about complex tori and theta functions (cf. Weil [9]). We shall write our complex torus $B$ in the form $B=C^{m} / \Delta$ with $m=$ $n+1$ and $\Delta$ a lattice of $\boldsymbol{C}^{m}$, and regard $\boldsymbol{C}^{m}$ as $\boldsymbol{R}^{2 m}$ with complex structure $J$. Let $H=\left(h_{k j}\right)$ be an $m \times m$ Hermitian matrix, and $H(u, v)=\sum h_{k j} u^{k} \bar{v}^{j}$ the corresponding Hermitian form. The imaginary part $A(u, v)$ of $H(u, v)$ is a skew symmetric bilinear form defined on $\boldsymbol{R}^{2 m} \times \boldsymbol{R}^{2 m}$ such that $A(J u, J v)=$ $A(n, v)$. We say that $A$ is intgral if $A$ takes integral values on $\Delta \times \Delta$. If $A$ is integral, there exists a basis $\left\{u_{1}, u_{1}^{\prime}, \cdots, u_{m}, u_{m}^{\prime}\right\}$ of $\Delta$ such that

$$
A(x, y)=\sum_{k=1}^{m} e_{k}\left(x^{k} y^{\prime k}-x^{\prime k} y^{k}\right)
$$

where $x=\sum x^{k} u_{k}+\sum x^{\prime k} u_{i c}^{\prime}, \quad y=\sum y^{k} u_{k}+\sum y^{\prime k} u_{k}^{\prime}$, and the nonnegative integers $e_{k}$ satisfy $e_{1}\left|e_{2}\right| \cdots \mid e_{m}$. (it is possible that $e_{p+1}=\cdots=e_{m}=0$ for some $1 \leq p \leq m$ ). We call $e_{1}, \cdots, e_{m}$ the elementary divisors of $A$ or of $H$. They are determined uniquely by $A$ and hence by $H$.

A holomorphic function $\theta$ on $C_{m}$ is called a theta function of type $(H, \Psi)$ if

$$
\theta(z+\sigma)=j(z, \sigma) \theta(z)
$$

for all $z \in C^{m}$ and $\sigma \in \Delta$, where the automorphic factor $j(z, \sigma)$ is of the form

$$
j(z, \sigma)=\Psi(\sigma) e\left[\frac{1}{2 i} H(z, \sigma)+\frac{1}{4 i} H(\sigma, \sigma)\right],
$$

where $\boldsymbol{e}=\exp 2 \pi i, H(u, v)$ is a Hermitian form on $\boldsymbol{C}^{m} \times \boldsymbol{C}^{m}$ whose imaginary part $A(u, v)$ is integral, and $\Psi$ is a map of $\Delta$ into $C^{*}$ satisfying $\Psi\left(\sigma+\sigma^{\prime}\right)$ $\cdot \boldsymbol{e}\left(\frac{1}{2} A\left(\sigma, \sigma^{\prime}\right)\right)=\Psi(\sigma) \Psi\left(\sigma^{\prime}\right)$ for any $\sigma$ and $\sigma^{\prime}$ in $\Delta$. We call an "automorphic 
factor" of the form (2.2) a theta factor of type $(H, \Psi)$. A holomorphic theta function $\theta$ on $C^{m}$ determines a divisor on $B$ in the following way. Let $\left\{U_{\alpha}\right)_{\alpha \in A}$ be an open covering of $B$ such that each connected component of $\pi^{-1}\left(U_{\alpha}\right)$ $\left(\pi: C^{m} \rightarrow B\right)$ is mapped homeomorphically onto $U_{\alpha}$, and let $\tilde{U}_{\alpha}$ be one of the connected components of $\pi^{-1}\left(U_{\alpha}\right)$. Then $\pi^{-1}\left(U_{\alpha}\right)=\bigcup_{o \in \Delta}\left(\sigma+\tilde{U}_{\alpha}\right)$. Let $\rho_{\alpha}=$ $\left(\pi \mid \tilde{U}_{\alpha}\right)^{-1}$. Then $\left\{\theta_{\alpha}\right\}_{\alpha \in A}$, where $\theta_{\alpha}=\theta \circ \rho_{\alpha}$ for each $\alpha \in A$, defines a positive divisor $D$ on $B$, which we shall denote by $(\theta)$ and is called the divisor of the theta function $\theta$. Conversely, every positive divisor $D$ is defind by a holomorphic theta function $\theta$ of type $(H, \Psi)$ for some $(H, \Psi)$, and the Chern class of the holomorphic line bundle $\{D\}$ is represented by the invariant $(1,1)$-form $h_{D}$ of the form

$$
h_{D}=\frac{1}{2} i \sum h_{k j} \zeta^{k} \wedge \bar{\zeta}^{j},
$$

where $H=\left(h_{k j}\right)$, and $\zeta^{1}, \cdots, \zeta^{m}$ denote, as in $\S 1$, holomorphic 1 -forms on $B$ such that $\pi^{*} \zeta^{k}=d w^{k},\left\{w^{1}, \cdots, w^{m}\right\}$ being the standard coordinates in $C^{m}$. It is known that if $D$ is a positive divisor, then $H$ is positive, and hence $c(\{D\})$ $\geq 0$ (see [9, Prop. 5, Chap. IV, No. 5]).

Let $b \in B$, and $D$ be a positive divisor. Then the divisor $T_{b} D$ is defined in an obvious way. Let $\Gamma$ be the group of all $b \in B$ such that $T_{b} D=D$. Then the null space $E_{0}$ of the Hermitian form $H$ associated to $D$ has the following properties: Since the intersection $\Delta_{0}=E_{0} \cap \Delta$ is a lattice of $E_{0}$, the image $\pi\left(E_{0}\right)$ of $E_{0}$ in $B$ is a complex subtorus isomorphic to $E_{0} / \Delta_{0}$ and $\pi\left(E_{0}\right)$ is a subgroup of finite index in the group $\Gamma$ (see Weil [9, Cor. 3, Chap. IV, No. 5]).

Let us apply these results to our divisor $D(X)$ defined by $X=\Phi(M)$. The Chern class $c(\{X\})$ of the line bundle $\{X\}$ is represented by a $(1,1)$-form $h_{\Phi}$ of the form

$$
h_{\Phi}=\frac{1}{2} i \sum h_{k j} \zeta^{k} \wedge \bar{\zeta}^{j},
$$

and we shall denote by $H_{\Phi}$ the Hermitian matrix $\left(h_{k j}\right)$ and also by the same letter the associated Hermitian form on $C^{m} \times C^{m}(m=n+1)$. The null space of $H_{\Phi}$ will be denoted by $E_{0}$. Then $\pi\left(E_{0}\right)$ is a complex subtorus of $B$ and is a subgroup of finite index of the group of all $b \in B$ such that $T_{b} D(X)=D(X)$. If $T_{b} D(X)=D(X)$, then we have $T_{b} X=X$, because $X=|D(X)|$, where $|D|$ denotes the carrier of a divisor $D$. Conversely, let $T_{b} X=X$. Clearly $\left|T_{b} D(X)\right|$ $=T_{b}|D(X)|=|D(X)|$. The divisor $T_{b} D(X)$ is positive and irreducible together with $D(X)$, and they have the same carrier. Then $D(X)$ and $T_{b} D(X)$ coincide (cf. for example [9, Appendix].) Therefore $\pi\left(E_{0}\right)$ is a subgroup of finite index of the group $\Gamma$ of all $b \in B$ such that $T_{b} X=X$. This combined with Lemma $2, \S 1$ gives $\Phi^{\prime}\left(\operatorname{Aut}_{0}(M)\right)=\pi\left(E_{0}\right)$. Since $\Phi^{\prime}$ and $\pi$ are both local isomorphisms (cf. Lemma 1, § 1), we obtain

Theorem 1. Let $\Phi: M \rightarrow B$ be a holomorphic immersion of an n-dimensional compact connected complex manifold $M$ into an $(n+1)$-dimensional 
complex torus $B$. Then the complex dimension of the complex torus $\mathrm{Aut}_{0}(M)$ is equal to the nullity of the Hermitian form $H_{\Phi}$ on $C^{n+1} \times C^{n+1}$ associated to $\Phi$. Moreover, the Chern class $c(\{X\})$ of the line bundle $\{X\}(X=\Phi(M))$ is represented by the $(1,1)$-form

$$
h_{\Phi}=\frac{1}{2} i \sum h_{k j} \zeta^{k} \wedge \bar{\zeta}^{j},
$$

where $H_{\Phi}=\left(h_{k j}\right)$, and $H_{\Phi}$ and hence $c(\{X\})$ are positive definite if and only if $\operatorname{Aut}_{0}(M)$ is trivial.

3. Let $A$ be the imaginary part of the Hermitian form $H_{\Phi}$. Then $A$ is integral, and let $\left(e_{1}, \cdots, e_{n+1}\right)$ be the elementary divisor of $A$. Let $S$ be the singular set of $X$. Then $\Phi: M-\Phi^{-1}(S) \rightarrow X-S$ is a proper holomorphic map of the $n-$ dimensional complex manifold $M-\Phi^{-1}(S)$ onto the $n$-dimensional complex manifold $X-S$, and for any $2 n$-form $\eta$ of compact carrier defined on $X-S$ we have

$$
\int_{M-\Phi-1(S)} \Phi^{*} \eta=d_{\Phi} \int_{X-S} \eta,
$$

where $d_{\Phi}$ denotes the degree of $\Phi: M-\Phi^{-1}(S) \rightarrow X-S$ (see Sternberg [8]). On the other hand, $X$ may be regarded as a $2 n$-cycle in $B$, and the integral

$$
\int_{X} \Psi
$$

over $X$ of an $2 n$-form $\Psi$ on $B$ is defined. It follows from the definition of the integral over $X$ (see Lelong [5]) and from (3.1) that

$$
\int_{M} \Phi^{*} \Psi=d_{\Phi} \int_{X} \Psi
$$

for any $2 n$-form $\Psi$ on $B$.

On the other hand, $c(\{X\})$ is the Poincare dual of the homology class of $X$, that is,

$$
\int_{X} \Psi=\int_{B} h_{\Phi} \wedge \Psi
$$

holds for any $2 n$-form $\Psi$ on $B$ (see Kodaira-Spencer [4, a] and Hirzebruch [1]). Letting $\Psi=h_{\Phi}^{n}$ in (3.2) and (3.3) we obtain

$$
\int_{M}\left(\Phi^{*} h_{\Phi}\right)^{n}=d_{\Phi} \int_{B} h_{\Phi}^{n+1}
$$

Put $X^{\prime}=X-S$ and $M^{\prime}=M-\Phi^{-1}(S)$, and let $i_{X^{\prime}}$ and $i_{M^{\prime}}$ be the inclusion maps of $X^{\prime}$ and $M^{\prime}$ into $B$ and $M$ respectively. We also denote by $\Phi^{\prime}$ the map $M^{\prime} \rightarrow X^{\prime}$ induced by $\Phi$. Then $\Phi \circ i_{M^{\prime}}=i_{X^{\prime}} \circ \Phi^{\prime}$. 
The line bundle $\Phi^{*}\left(i_{X^{\prime}}^{*}\{X\}\right)$ over $M^{\prime}$ is the restriction of the normal bundle $N$ of $M$ with respect to the holomorphic immersion $\Phi$ of $M$ into $B$, and hence $i_{M^{\prime}}^{*}, N=\Phi^{*}\left(i_{X^{\prime}}^{*}\{X\}\right)$, and $i_{M^{\prime}}^{*}\left(\Phi^{*} h_{\Phi}\right)^{n}$ represents $i_{M^{\prime}}^{*} c(N)^{n}$. Since $\Phi^{-1}(S)$ is an analytic subset of $M$ and $M^{\prime}=M-\Phi^{-1}(S)$, we have

$$
\int_{M}\left(\Phi^{*} h_{\Phi}\right)^{n}=\int_{M^{\prime}} i_{M^{\prime}}^{*}\left(\Phi^{*} h_{\Phi}\right)^{n}=\int_{M^{\prime}} i_{M^{\prime}}^{*} c(N)^{n}=\int_{M} c(N)^{n}
$$

We have an exact sequence

$$
0 \rightarrow T(M) \rightarrow \Phi^{*} T(B) \rightarrow N \rightarrow 0,
$$

and $\Phi^{*} T(B)$ is a trivial vector bundle of fibre dimension $n+1$ since $B$ is a complex torus. Thus we have

$$
\left(1+c_{1}(M)+\cdots+c_{n}(M)\right)(1+c(N))=1,
$$

from which it follows that

$$
c_{k}(M)=(-1)^{k} c(N) \quad(k=1,2, \cdots, n),
$$

where $c_{k}(M)$ denotes the $k$-th Chern class of $M$. In particular $c(N)^{n}=$ $(-1)^{n} c_{n}(M)$, and we have

$$
\int_{M} c(N)^{n}=(-1)^{n} E(M),
$$

where $E(M)$ denotes the Euler number of $M$. It follows then from (3.4) and (3.5) that

$$
E(M)=(-1)^{n} d_{\Phi} \int_{B} h_{\Phi}^{n+1}
$$

To compute the integral on the right hand side of (3.7), we notice

$$
\int_{B} h_{\Phi}^{n+1}=\int_{F}\left(\pi^{*} h_{\Phi}\right)^{n+1},
$$

where $F$ is a fundamental domain of $\Delta$ and $\pi^{*} h_{\Phi}=\frac{1}{2} i \sum h_{k j} d w^{k} \wedge d \bar{w}^{j}$. Moreover,

$$
H_{\emptyset}(u, v)=A(J u, v)+i A(u, v),
$$

where $\boldsymbol{J}$ is the complex structure in $\boldsymbol{R}^{2(n+1)}$ defining $\boldsymbol{C}^{n+1}$, the real skewsymmetric form $A$ may be regarded as a 2 -form on $\boldsymbol{R}^{2(n+1)}$, and

$$
\pi^{*} h_{\oplus}=-A
$$


as 2-forms on $\boldsymbol{R}^{2(n+1)}$. Let $\left\{u_{1}, u_{1}^{\prime}, \cdots, u_{n+1}, u_{n+1}^{\prime}\right\}$ be a basis of $\Delta$ such that $A\left(u_{k}, u_{j}^{\prime}\right)=e_{k} \delta_{k j}$ and $A\left(u_{k}, u_{j}\right)=A\left(u_{k}^{\prime}, u_{j}^{\prime}\right)=0(k, j=1,2, \cdots, n+1)$, and let $\left\{x^{1}, x^{\prime 1}, \cdots, x^{n+1}, x^{\prime n+1}\right\}$ be the corresponding coordinates in $R^{2(n+1)}$. Then

$$
\begin{gathered}
A=\sum_{k} e_{k} d x^{k} \wedge d x^{\prime k} \\
\frac{1}{(n+1) !} A^{n+1}=\left(e_{1} \cdots e_{n+1}\right) d x^{1} \wedge d x^{\prime 1} \wedge \cdots \wedge d x^{n+1} \wedge d x^{\prime n+1} .
\end{gathered}
$$

Since a fundamental domain $F$ of $\Delta$ is given by

$$
F=\left\{\left(x^{1}, x^{\prime 1}, \cdots, x^{n+1}, x^{\prime n+1}\right) \mid 0 \leq x^{k}, x^{\prime k} \leq 1, k-1,2, \cdots, n+1\right\},
$$

we get

$$
\int_{F} A^{n+1}= \pm(n+1) !\left(e_{1} \cdots e_{n+1}\right)
$$

where the sign depends on the orientation of the coordinates $\left\{u_{1}, u_{1}^{\prime}, \cdots, u_{n+1}\right.$, $\left.u_{n+1}^{\prime}\right\}$ of $\boldsymbol{R}^{2(n+1)}$. It follows from (3.8), (3.9) and (3.10) that the absolute value of the integral $\int_{B} h_{\Phi}^{n+1}$ is equal to $(n+1) !\left(e_{1} \cdots e_{n+1}\right)$. However, the matrix $H_{\Phi}$ is positive so that $2(n+1)$-form $h_{\Phi}^{n+1}$ should be nonnegative. Thus the value of the integral should also be nonnegative, and we get

$$
\int_{B} h_{\Phi}^{n+1}=(n+1) !\left(e_{1} \cdots e_{n+1}\right) \text {. }
$$

From (3.7) we obtain the formula

$$
E(M)=(-1)^{n}(n+1) ! d_{\Phi}\left(e_{1} \cdots e_{n+1}\right),
$$

where the positive integer $d_{\Phi}$ is the degree of the map $\Phi: M-\Phi^{-1}(S) \rightarrow$ $X-S$, and $\left(e_{1}, \cdots, e_{n+1}\right)$ are elementary divisors of the imaginary part $A$ of the Hermitian form $H_{\phi}$. Since the elementary divisors are all positive if and only if $A$ and hence $H_{\Phi}$ are nondegenerate, we obtain

Theorem 2. Let $\Phi: M \rightarrow B$ be a holomorphic immersion of an n-dimensional compact connected complex manifold $M$ into an $(n+1)$-dimensional complex torus $B$. Then the following three conditions are equivalent:

1) $\mathrm{Aut}_{0} M=\{1\}$,

2) $H_{\Phi}$ is positive definite, or, equivalently, the Chern class $c(\{X\})$ of the holomorphic line bundle $\{X\}$ over $B$ is positive definite,

3) the Euler number $E(M)$ of $M$ is not zero.

Since the pullback of $c(\{X\})$ by $\Phi$ defines a Hodge form on $M$, we obtain from Kodaira's theorem the following corollary which is a special case of a more general theorem proved in [6]. 
Corollary. Let $M$ be an n-dimensional compact connected complex manifold admitting a holomorphic immersion into an $(n+1)$-dimensional complex torus. If $E(M) \neq 0$, then $M$ is algebraic.

Let us consider now the quotient $N=M / \operatorname{Aut}_{0}(M)$. Then by $\S 1, N$ admits a holomorphic immersion into $B^{\prime}=B / C$, where $C=\Phi^{\prime}\left(\operatorname{Aut}_{0}(M)\right)$. However $\operatorname{dim} C=\operatorname{dim} \operatorname{Aut}_{0}(M)$ by Lemma 1 , and hence $\operatorname{dim} B^{\prime}=\operatorname{dim} N+1$. Since $\operatorname{Aut}_{0}(N)=\{1\}$ by Proposition 1 , from Theorem 2 and its corollary we get

Theorem 3. Let $M$ be an $n$-dimensional compact connected complex manifold admitting a holomorphic immersion in an $(n+1)$-dimensional complex torus $B$. Then $M$ is a principal fibre bundle over the quotient manifold $N=$ $M / \operatorname{Aut}_{0}(M)$ of the structure group $\operatorname{Aut}_{0}(M)$, and $N$ has the following properties:

a) $N$ is algebraic, and $N$ admits a holomorphic immersion $\Psi$ into a complex torus $B^{\prime}$ with $\operatorname{dim} B^{\prime}=\operatorname{dim} N+1$.

b) $E(N) \neq 0$, and the Chern class $c\left(\left\{X^{\prime}\right\}\right)$ of $\left\{X^{\prime}\right\}$ is positive definite, where $\left\{X^{\prime}\right\}$ denotes the holomorphic line bundle defined by the divisor associated with the image $X^{\prime}=\Psi(N)$ of $N$ in $B^{\prime}$.

Remark. It follows from a recent result of Nagano-Smyth [7] that, without assuming that $\operatorname{dim} B=n+1, \operatorname{Aut}_{0}(M)=\{1\}$ if and only if the Ricci tensor of the Kähler metric on $M$, which is induced from a flat Kähler metric on $B$, is negative definite almost everywhere (it is always negative everywhere). Thus the Chern number $c_{1}^{n}$ of $M$ is nonzero, and $N$ is algebraic by a theorem proved in [6].

4. We shall prove the following theorem.

Theorem 4. Let $M$ be an n-dimensional compact connected Kähler manifold. Assume that the Euler number $E(M)$ of $M$ is not zero and $n \geq 2$. Then $M$ admits a holomorphic immersion into an $(n+1)$-dimensional complex torus if and only if the following two conditions are satisfied:

1) the cotangent bundle $T^{*}(M)$ of $M$ is ample,

2) $h^{1,0}(M)=n+1$, where $h^{1,0}(M)$ is the number of linearly independent holomorphic 1-forms on $M$.

Proof. If the above two conditions are verified, then without assuming that $n \geq 2$ and $E(M) \neq 0$ we see that the canonical map $J$ from $M$ into the Albanese variety of $M$ is a holomorphic immersion as we have already observed in $\S 1$.

Suppose now that $M$ has a holomorphic immersion $\Phi$ into a complex torus $B$ of dimension $n+1$. Then $T^{*}(M)$ is ample (see $\S 1$ ), and the condition 2 ) follows from the following lemma if we assume $E(M) \neq 0$ and $n \geq 2$.

Lemma 3. Assume that $E(M) \neq 0$, and $M$ admits a holomorphic immersion into a complex torus $B$ of dimension $n+1$, where $n=\operatorname{dim} M$. Then we have

$$
(n+1) h^{0, q}(M)=h^{1, q}(M) \quad(q=0,1, \cdots, n-2)
$$


In particular, $h^{1,0}(M)=n+1$ for $n \geq 2$.

Let $N$ be the normal bundle of $M$ with respect to the immersion $\Phi: M \rightarrow B$. Then we have

$$
0 \rightarrow T(M) \rightarrow I_{n+1} \rightarrow N \rightarrow 0,
$$

where $I_{n+1}=\Phi^{*} T(B)$ denotes the trivial vector bundle of fibre dimension $n+1$. Then we get

$$
0 \rightarrow N^{*} \rightarrow I_{n+1} \rightarrow T^{*}(M) \rightarrow 0
$$

and hence the exact sequence of cohomologies:

$$
\rightarrow H^{q}\left(M, N^{*}\right) \rightarrow H^{q}\left(M, \mathfrak{o}^{n+1}\right) \rightarrow H^{q}\left(M, T^{*}(M)\right) \rightarrow H^{q+1}\left(M, N^{*}\right) \rightarrow .
$$

Let us consider the Chern class $c(N)$ of $N$. As we have seen in $\S 3, N$ and $\Phi^{*}\{X\}$ coincide on $M^{\prime}=M-\Phi^{-1}(S)$. Let $F=N \cdot \Phi^{*}\{X\}^{-1}$, and for each small $\varepsilon>0$ let $U_{\varepsilon}$ be an open neighborhood of $\Phi^{-1}(S)$ such that the measure (with respect to any Kähler metric of $M$ ) of $U_{\varepsilon}$ tends to 0 as $\varepsilon$ tends to 0 . For each $\varepsilon, c(F)$ is represented by a $(1,1)$-form $\eta_{\varepsilon}$ which is 0 outside $U_{\varepsilon}$. Since $N=$ $\Phi^{*}\{X\} \cdot F, c(N)$ is represented by $\Phi^{*} h_{\Phi}+\eta_{\varepsilon}$. Since $E(M) \neq 0, h_{\Phi}$ and hence $\Phi^{*} h_{\Phi}$ are positive definite by Theorem 2 . Under this situation Kodaira's original proof of his vanishing theorem (see Kodaira [3]) works well, and we can conclude that $H^{q}\left(M, N^{*}\right)=0$ for $q<n$. It thus follows from the above exact sequence of cohomologies that $H^{q}\left(M, \mathfrak{o}^{n+1}\right) \cong H^{q}\left(M, T^{*}(M)\right)$ for $q+1<n$, and this proves our lemma and at the same time Theorem 4.

Remark. The assumption $n \geq 2$ is necessary. In fact, we can immerse a compact Riemann surface $M$ of genus $g>2$ for some $g$ into a 2-dimensional complex torus whereas $h^{1,0}(M)=g>2$.

Under the assumption in Theorem 4 we show that $\Phi$ induces an isomorphism of $H^{1,0}(B)$ onto $H^{1,0}(M)$. We know by Lemma 3 that $\operatorname{dim} H^{1,0}(M)=n+1$, and $H^{1,0}(B)$ is spanned by $\zeta^{1}, \cdots, \zeta^{n+1}$, so that $\operatorname{dim} H^{1,0}(B)=n+1$. Therefore it is sufficient to show that $\Phi^{*}: H^{1,0}(B) \rightarrow H^{1,0}(M)$ is injective. By a linear change of coordinates in $C^{n+1}$, we may assume that the kernel of $\Phi^{*}: H^{1,0}(B)$ $\rightarrow H^{1,0}(M)$ is spanned by $\zeta^{1}, \cdots, \zeta^{s}(s \geq 0)$. As in $\S 1$, let $\omega^{k}=\Phi^{*} \zeta^{k}$. Since $\omega^{1}=\cdots=\omega^{s}=0, \omega^{s+1}, \cdots, \omega^{n+1}$ should span the cotangent vector space of $M$ at each point of $M$. Therefore $s \leq 1$. If $s=1$, then $\omega^{2}, \cdots, \omega^{n+1}$ must be linearly independent at each point of $M$, and $M$ is parallizable, which contradicts the assumption that $E(M)$ is not zero. Let now $B=C^{n+1} / \Delta_{B}$, and $A=C^{n+1} / \Delta_{A}$ where $A$ denotes the Albanese variety of $M$. We denote by $\pi_{B}$ and $\pi_{A}$ the projections of $C^{n+1}$ onto $B$ and $A$ respectively. Then by $\S 1$

$$
\Phi(x)=T_{\Phi\left(x_{0}\right)}\left(\pi_{B}\left(\int_{x_{0}}^{x} \omega^{1}, \cdots, \int_{x_{0}}^{x} \omega^{n+1}\right)\right)
$$




$$
J(x)=\pi_{A}\left(\int_{x_{0}}^{x} \omega^{1}, \cdots, \int_{x_{0}}^{x} \omega^{n+1}\right)
$$

For any closed path $\gamma$ starting at $x_{0}$ we have

$$
\pi_{B}\left(\int_{r} \omega^{1}, \cdots, \int_{r} \omega^{n+1}\right)=\text { the identity element } e_{B} \text { of } B
$$

and

$$
\left(\int_{r} \omega^{1}, \cdots, \int_{r} \omega^{n+1}\right) \in \Delta_{B}
$$

which shows that $\Delta_{A} \subset \Delta_{B}$. Therefore there is a surjective homomorphism $f: A \rightarrow B$ such that $f\left(\pi_{A}\left(\int_{x_{0}}^{x} \omega^{1}, \cdots, \int_{x_{0}}^{x} \omega^{n+1}\right)\right)=\pi_{B}\left(\int_{x_{0}}^{x} \omega^{1}, \cdots, \int_{x_{0}}^{x} \omega^{n+1}\right)$. Thus we have shown that for any holomorphic immersion $\Phi$ of $M$ into an $(n+$ 1)-dimensional torus $B$ there exists a homomorphism $f$ of the Albanese variety $A$ of $M$ onto $B$ such that

$$
\Phi(x)=T_{\Phi\left(x_{0}\right)}(f(J(x)))
$$

for all $x \in M$.

Let $H^{1,1}(M, R)$ and $H^{1,1}(B, R)$ denote respectively the subspaces of the real de Rham cohomology group $H^{2}(M, R)$ and $H^{2}(B, R)$ whose elements are represented by closed real forms of type $(1,1)$. We show that $\Phi^{*}: H^{1,1}(B, R) \rightarrow$ $H^{1,1}(M, R)$ is bijective provided $n \geq 3$. Since $\operatorname{dim}_{R} H^{1,1}(B, R)=h^{1,1}(B)=$ $(n+1)^{2}$ and $\operatorname{dim}_{R} H^{1,1}(M, R)=h^{1,1}(M), h^{1,1}(M)=(n+1) h^{0,1}(M)=(n+1)^{2}$ by Lemma 3 provided $n \geq 3$. Therefore it is sufficient to show that $\Phi^{*}$ is injective. The space $H^{1,1}(B, R)$ is identified with the space of all $(1,1)$-forms on $B$ of the form $\theta=i \sum \theta_{k j} \zeta^{k} \wedge \bar{\zeta}^{k}$ where $\Theta=\left(\theta_{k j}\right)$ is a constant Hermitian matrix. Let $\omega^{k}=\Phi^{*} \zeta^{k}(k=1, \cdots, n+1)$, and suppose that $\Phi^{*} \theta \sim 0$. Then $\Theta$ cannot be (positive or negative) definite. In fact, if $\Theta$ is positive definite for example, then $\Phi^{*} \theta$ is the Kähler form of the Kähler metric $\sum_{k, j} \theta_{k j} \omega^{k} \bar{\omega}^{j}$ on $M$, and $\Phi^{*} \theta$ cannot be cohomologous to zero. After a suitable linear change of coordinates in $C^{n+1}$, we may assume that $\Theta$ is of the form

$$
\Theta=\left(\begin{array}{ccc}
O_{k} & & \\
& E_{j} & \\
& & -E_{l}
\end{array}\right) \quad(k+j+l=n+1),
$$

where $O_{k}$ denotes the $k \times k$ zero matrix, and $E_{j}$ and $E_{l}$ are the unit matrices of types $j \times j$ and $l \times l$ respectively. We may assume $j \leq l$ (otherwise replace $\theta$ by $-\theta)$. To simplify our notation, put $\zeta_{k}=\zeta^{k} \wedge \bar{\zeta}^{k}, \omega_{k}=\omega^{k} \wedge \bar{\omega}^{k}, \omega=$ $i \sum \omega_{k}$. Then $\theta=i\left(\zeta_{k+1}+\cdots+\zeta_{k+j}-\zeta_{k+j+1}-\cdots-\zeta_{n+1}\right)$, and $\omega+\Phi^{*} \theta$ $=\omega^{\prime}$ where $\omega^{\prime}=i\left(\omega_{1}+\cdots+\omega_{k}+2 \omega_{k+1}+\cdots+2 \omega_{k+j}\right)$. Since $\Phi^{*} \theta \sim 0$, 
$\omega \sim \omega^{\prime}$ and hence $\omega^{n} \sim \omega^{\prime n}$. If $k+j<n$, then $\omega^{\prime n}=0$ and $\omega^{n} \sim 0$, and this is impossible because $\omega$ is the Kähler form of the Kähler metric $\sum \omega^{k} \cdot \bar{\omega}^{k}$. Therefore $k+j=n$ or $k+j=n+1$. Suppose $k+j=n$. Since $k+j+$ $l=n+1$, we get $l=1$, and as we have assumed that $j \leq l, j$ is either equal to 0 or 1 . If $j=0$, we have $k=n$, and

$$
\Phi^{*} \theta=-i \omega^{n+1} \wedge \bar{\omega}^{n+1} \sim 0, \quad \omega \sim \omega^{\prime}=i\left(\omega_{1}+\cdots+\omega_{n}\right) .
$$

If $j=1$, we have $k=n-1, l=j=1$ and

$$
\Phi^{*} \theta=i\left(\omega_{n}-\omega_{n+1}\right) \sim 0, \quad \omega \sim \omega^{\prime}=i\left(\omega_{1}+\cdots+\omega_{n-1}+2 \omega_{n}\right) .
$$

If $k+j=n+1$, then $l=0$, and hence $j=0$ and $\Theta=0$ which implies $\theta=0$.

In either of the cases (A) and (B), we have $\omega^{n} \sim \omega^{\prime n}$ and

$$
\int_{M} \omega^{n}=\int_{M} \omega^{\prime n}
$$

with $\omega^{n}=\Phi^{*}\left(i \sum_{k=1}^{n+1} \zeta_{k}\right)^{n}$ and either $\omega^{\prime}=\Phi^{*}\left(i \sum_{k=1}^{n} \zeta_{k}\right)^{n}$ (for case (A)) or $\omega^{\prime n}=\Phi^{*}\left(i \sum_{k=1}^{n-1} \zeta_{k}+2 i \zeta_{n}\right)^{n}$ (for case (B)). Put $\zeta=i \sum_{k=1}^{n+1} \zeta_{k}$ and $\zeta^{\prime}=$ $i \sum_{k=1}^{n} \zeta_{k}$ (for case (A)) or $\zeta^{\prime}=i \sum_{k=1}^{n-1} \zeta_{k}+2 i \zeta_{n}$ (for case (B)). Then by (3.2) and (4.1)

$$
\int_{X} \zeta^{n}=\int_{X} \zeta^{\prime n}
$$

where $X=\Phi(M)$. Since $c(\{X\})$ is the Poincare dual of the homology class $X$, we get

$$
\int_{B} h_{\Phi} \wedge \zeta^{n}=\int_{B} h_{\Phi} \wedge \zeta^{\prime n}
$$

and hence $h_{\Phi} \wedge \zeta^{n}=h_{\Phi} \wedge \zeta^{\prime n}$ because both sides are invariant and of type $(n, n)$. As in $\S 3$, let $h_{\Phi}=\frac{1}{2} i \sum h_{k j} \zeta^{k} \wedge \bar{\zeta}^{j}$. Since $E(M) \neq 0$, the Hermitian matrix $H_{\Phi}=\left(h_{k j}\right)$ is positive definite by Theorem 2 , and $\zeta^{n}=i^{n} n ! \sum_{k=1}^{n+1} \zeta_{1} \wedge$ $\cdots \wedge \hat{\zeta}_{k} \wedge \cdots \wedge \zeta_{n+1}, h_{\Phi} \wedge \zeta^{n}=\frac{1}{2} i^{n+1} n ! \operatorname{Tr} H_{\Phi}\left(\zeta_{1} \wedge \cdots \wedge \zeta_{n+1}\right)$. Suppose that the case (A) occurs. Then $\zeta^{\prime n}=i^{n} n ! \zeta_{1} \wedge \cdots \wedge \zeta_{n}$, and $h_{\Phi} \wedge \zeta^{\prime n}=$ $\frac{1}{2} i^{n+1} n ! h_{n+1, n+1}\left(\zeta_{1} \wedge \cdots \wedge \zeta_{n+1}\right)$. It follows from $h_{\Phi} \wedge \zeta^{n}=h_{\Phi} \wedge \zeta^{\prime n}$ that $\sum_{k=1}^{n} h_{k k}=0$. However $n \times n$ Hermitian matrix $H^{\prime}=\left(h_{k j}\right)(1 \leq k, j \leq n)$ is positive definite, and it is impossible that $\operatorname{Tr} H^{\prime}=0$, so the case (A) cannot occur. Suppose now that the case (B) occurs. Then $\zeta^{\prime}=i\left(\sum_{k=1}^{n-1} \zeta_{k}+2 \zeta_{n}\right)$, $\zeta^{\prime n}=i^{n} n ! 2\left(\zeta_{1} \wedge \cdots \wedge \zeta_{n}\right)$, and $h_{\Phi} \wedge \zeta^{\prime n}=i^{n+1} n ! h_{n+1, n+1}\left(\zeta_{1} \wedge \cdots \wedge \zeta_{n+1}\right)$. It follows from $h_{\Phi} \wedge \zeta^{n}=h_{\Phi} \wedge \zeta^{\prime n}$ that $\operatorname{Tr} H_{\Phi}=2 h_{n+1, n+1}$. In the case (B), $\omega_{n} \sim \omega_{n+1}$, and since each $\omega_{k}$ is closed, we have also $\omega_{1} \wedge \cdots \wedge \omega_{n-1} \wedge \omega_{n}$ $\sim \omega_{1} \wedge \cdots \wedge \omega_{n-1} \wedge \omega_{n+1}$ and hence 


$$
\int_{M} \omega_{1} \wedge \cdots \wedge \omega_{n-1} \wedge \omega_{n}=\int_{M} \omega_{1} \wedge \cdots \wedge \omega_{n-1} \wedge \omega_{n+1}
$$

From this we obtain as before $h_{\Phi} \wedge \zeta_{1} \wedge \cdots \wedge \zeta_{n-1} \wedge \zeta_{n}=h_{\Phi} \wedge \zeta_{1} \wedge \cdots \wedge$ $\zeta_{n-1} \wedge \zeta_{n+1}$, and $h_{n+1, n+1} \zeta_{1} \wedge \cdots \wedge \zeta_{n+1}=h_{n, n} \zeta_{1} \wedge \cdots \wedge \zeta_{n+1}$ which implies $h_{n+1, n+1}=h_{n, n}$. Combining this with $\operatorname{Tr} H_{\Phi}=2 h_{n+1, n+1}$ we get $\sum_{k=1}^{n-1} h_{k k}$ $=0$, and this contradicts the fact that the $(n-1) \times(n-1)$ Hermitian matrix $\left(h_{k j}\right)(1 \leq k, j \leq n-2)$ is also positive definite. Thus both cases (A) and (B) cannot occur, and therefore $\theta$ should be equal to 0 ; this concludes our proof.

It is also shown that every cohomology class in $H^{1,1}(M, R)$ is represented by a unique $(1,1)$-form of the type

$$
i \sum \theta_{k j} \omega^{k} \wedge \bar{\omega}^{j}
$$

where $\left(\theta_{k j}\right)$ is a constant Hermitian matrix of type $(n+1) \times(n+1)$, and $\left\{\omega^{1}, \cdots, \omega^{n+1}\right\}$ with $\omega^{k}=\Phi^{*} \zeta^{k}$ is a basis of the space of holomorphic 1 -forms on $M$. From this and a theorem of Weil it follows that every divisor on $M$ is a divisor of a theta function on the universal covering manifold $\tilde{M}$ of $M$ (see Weil [9, Th. 2 on P. 99]).

Summing up and combining with Theorem 4 we obtain

Theorem 5. Let $M$ be a compact connected Kähler manifold such that $T^{*}(M)$ is ample, $E(M) \neq 0$ and $h^{1,0}(M)=\operatorname{dim} M+1$. Then the canonical map $J: M \rightarrow A$ of $M$ into the Albanese variety is an immersion. Moreover, if $n=\operatorname{dim} M \geq 3$, then, $J$ induces a bijection of $H^{1,1}(A, R)$ onto $H^{1,1}(M, R)$, every cohomology class of $H^{1,1}(M, R)$ is represented by a unique $(1,1)$-form of the type $\theta=i \sum \theta_{k j} \omega^{k} \wedge \bar{\omega}^{j}$, where $\left\{\omega^{1}, \cdots, \omega^{n+1}\right\}$ is a basis of the space of holomorphic 1-forms on $M$ and $\left(\theta_{k j}\right)$ is a constant Hermitian matrix and every divisor of $M$ is the divisor of a theta function on the universal covering manifold $\tilde{M}$ of $M$.

5. In this section we always denote by $M$ an $n$-dimensional compact connected Kähler manifold admitting a holomorphic immersion into an $(n+1)$ dimensional complex torus, and assume that $E(M) \neq 0$. Then the canonical map $J: M \rightarrow A$ of $M$ into the Albanese variety $\mathrm{A}$ is an immersion, and $A$ is $(n+1)$-dimensional. The Chern class $c(\{X\})$ of the line bundle $\{X\}$ associated to $X=J(M)$ is positive definite, and is represented by a unique $(1,1)$-form

$$
h_{J}=\frac{1}{2} i \sum h_{k j} \zeta^{k} \wedge \bar{\zeta}^{j}
$$

Here the constant Hermitian matrix $H_{J}$ is positive definite, and the imaginary part A of the associated Hermitian form is integral valued on $\Delta_{A} \times \Delta_{A}$ where we write $A=C^{n+1} / \Delta_{A}$. The elementary divisors of $A$ (or $H_{J}$ ) denoted by $e_{1}, \cdots, e_{n+1}$ are all positive integers since $A$ is nondegenerate. For any $n$-tuple of integers $\pi=\left(p_{1}, \cdots, p_{n}\right)$ such that $\sum k p_{k}=n$, let

$$
c_{\pi}[M]=\int_{M} c_{1}^{p_{1}} \cdots c_{n}^{p_{n}}
$$


where $c_{k}$ denotes the $k$-th Chern class of $M$. These numbers are called the Chern numbers of $M$. By (3.6), $c_{1}^{p_{1}} \cdots c_{n}^{p_{n}}=(-1)^{n} c(N)^{n}$ for all $\pi$ so that the Chern numbers are all equal. Since $E(M)$ is equal to $c_{\pi}[M]$ with $\pi=$ $(0,0 \cdots, 1)$, every Chern number of $M$ is equal to $E(M)$ :

$$
E(M)=c_{\pi}[M] \quad \text { for any } \pi .
$$

Let $Y$ be another $n$-dimensional compact connected Kähler manifold with $E(Y) \neq 0$ admitting a holomorphic immersion in an $(n+1)$-dimensional complex torus. Then we also have $E(Y)=c_{\pi}[Y]$ for any $\pi$ and hence

$$
c_{\pi}[M]=\rho \cdot c_{\pi}[Y] \quad \text { for any } \pi,
$$

where $\rho=E(M) / E(Y)$ is a nonzero rational number.

We say that two $n$-dimensional compact connected Kähler manifolds $M$ and $Y$ are proportional if their Chern numbers satisfy (5.1) for some nonzero rational $\rho$ (cf. Hirzebruch [1]). It follows then from Riemann-Roch-Hirzebruch theorem that

$$
\chi(M)=\rho \cdot \chi(Y), \quad \chi\left(M, K_{M}^{r}\right)=\rho \cdot \chi\left(Y, K_{Y}^{r}\right),
$$

where $K_{M}$ and $K_{Y}$ denote the canonical bundles of $M$ and $Y$ respectively, and $r$ is an integer. We shall compute $\chi(M)$ and $\chi\left(M, K_{M}^{r}\right)$ by choosing $Y$ suitably.

Since $E(M) \neq 0, M$ is algebraic (Theorem 2, Cor.) and hence $A$ is algebraic. Let $Y$ be a nonsingular hyperplane section of $A$ (with respect to a projective imbedding of $A$ ). By Lefschetz theorem, $b_{p}(Y)=b_{p}(A)=\left(\begin{array}{c}2 n+2 \\ p\end{array}\right)$ for $p=$ $0,1, \cdots, n-1$ and $b_{n}(Y) \geq b_{n}(A)=\left(\begin{array}{c}2 n+2 \\ n\end{array}\right)$. By a computation using the Poincare dualty we see that

$$
E(Y)=(-1)^{n}\left\{b_{n}(Y)-b_{n}(A)+\frac{(2 n+2) !}{(n+1) !(n+2) !}\right\}
$$

so that $E(Y) \neq 0$, and the identity map of $Y$ into $A$ is an immersion (as a matter of fact, the identity map of $Y$ into $A$ is the canonical map, and $A$ is the Albanese variety of $Y$ ).

We shall denote by $\{Y\}$ the line bundle over $A$ associated with the nonsingular positive divisor $Y$ of $A$. The Chern class $c(\{Y\})$ of $\{Y\}$ is represented by a unique $(1,1)$-form of the type

$$
\theta_{Y}=\frac{1}{2} i \sum \theta_{k j} \zeta^{k} \wedge \bar{\zeta}^{j}
$$

where $H_{Y}=\left(\theta_{k j}\right)$ is a constant positive definite Hermitian matrix, and the imaginary part of the associated Hermitian form is integral valued on $\Delta_{A} \times \Delta_{A}$. 
We denote by $f_{1}, \cdots, f_{n+1}$ the elementary divisor of $H_{Y}$. As a special case of the formula (3.11) we get

$$
E(Y)=(-1)^{n}(n+1) !\left(f_{1} \cdots f_{n+1}\right) .
$$

We also obtain from (5.3) and (5.4)

$$
b_{n}(Y)=\frac{n(2 n+2) !}{(n+1) !(n+2) !}+(n+1) !\left(f_{1} \cdots f_{n+1}\right)
$$

We shall use the following two formulas:

1) Riemann-Roch-Hirzebruch formula for complex tori: For any holomorphic line bundle $F$ over $A$

$$
\chi(A, F)=\frac{1}{(n+1) !} \int_{A} c(F)^{n+1}
$$

(This formula follows easily from the Riemann-Roch-Hirzebruch theorem, because Chern classes of $A$ are all zero.)

2) Kodaira-Spencer formula $[4, b]$ : For any holomorphic line bundle $F$ over $A$ and for any nonsingular divisor $Y$ of $A$

$$
\chi(A, F)=\chi\left(A, F \otimes\{Y\}^{-1}\right)+\chi(Y, F \mid Y) .
$$

Let $F$ be the trivial line bundle over $A$. Then $\chi(A, F)=\chi(A)=$ $\sum_{q}(-1)^{q} h^{0, q}(A)=\sum(-1)^{q}\left(\begin{array}{l}n \\ q\end{array}\right)=0$. Hence $\chi(Y)=-\chi\left(A,\{Y\}^{-1}\right)$ by (5.6), and

$$
\chi\left(A,\{Y\}^{-1}\right)=\frac{(-1)^{n+1}}{(n+1) !} \int_{A} c(\{Y\})^{n+1}=(-1)^{n+1}\left(f_{1} \cdots f_{n+1}\right)
$$

by (5.5) as in $\S 3$, so that $\chi(Y)=(-1)^{n}\left(f_{1} \cdots f_{n+1}\right)$ which together with (5.4) gives

$$
\chi(Y) / E(N)=1 /(n+1) ! .
$$

It follows from (5.2), (5.7) and (3.11) that

$$
\chi(M)=\frac{E(M)}{E(Y)} \chi(Y)=\frac{E(M)}{(n+1) !}=(-1)^{n} d_{J}\left(e_{1} \cdots e_{n+1}\right)
$$

where $e_{1}, \cdots, e_{n+1}$ are the elementary divisors of $H_{J}$, and $d_{J}$ is the degree of the map $J: M-J^{-1}(S) \rightarrow X-S, S$ denoting the singularities of $X=J(M)$. On the other hand 


$$
\begin{aligned}
\chi(Y) & =\sum_{q=1}^{n}(-1)^{b} h^{0, q}(Y)=\sum_{q=0}^{n-1}(-1)^{q}\left(\begin{array}{c}
n+1 \\
q
\end{array}\right)+(-1)^{n} h^{0, q}(Y) \\
& =-(-1)^{n}\left(\begin{array}{c}
n+1 \\
n
\end{array}\right)-(-1)^{n+1}+(-1)^{n} h^{0, n}(Y) .
\end{aligned}
$$

We have shown that $\chi(Y)=(-1)^{n}\left(f_{1} \cdots f_{n+1}\right)$ so that

$$
h^{0, n}(Y)=n+f_{1} \cdots f_{n+1} .
$$

Now putting $F=\{Y\}^{r}$ in (5.6) gives $\chi\left(A,\{Y\}^{r}\right)=\chi\left(A,\{Y\}^{r-1}\right)+\chi\left(Y, N^{r}\right)$, where $N=\{Y\} \mid Y$ is the normal bundle of $Y$. By (5.5) we get

$$
\begin{aligned}
\chi\left(Y, N^{r}\right) & =\frac{1}{(n+1) !} \int_{A}\left(r^{n+1}-(r-1)^{n+1}\right) c(\{Y\})^{n+1} \\
& =\left(r^{n+1}-(r-1)^{n+1}\right) f_{1} \cdots f_{n+1}
\end{aligned}
$$

On the other hand $c\left(K_{Y}\right)=-c_{1}(Y)=c(N)$, and from the Riemann-RochHirzebruch theorem for $Y$ it follows that $\chi\left(Y, N^{r}\right)=\chi\left(Y, K_{Y}^{r}\right)$ so that $\chi\left(Y, K_{Y}^{r}\right)$ $=\left(r^{n+1}-(r-1)^{n+1}\right) f_{1} \cdots f_{n+1}=(-1)^{n}\left(r^{n+1}-(r-1)^{n+1}\right) E(Y) /(n+1) !$. Thus $\chi\left(Y, K_{Y}^{r}\right) / E(Y)=(-1)^{n}\left(r^{n+1}-(r-1)^{n+1}\right) /(n+1) !, \quad$ and from $\chi\left(M, K_{M}^{r}\right)=(E(M) / E(Y)) \chi\left(Y, K_{Y}^{r}\right)$ we obtain the following formula :

$$
\chi\left(M, K_{M}^{r}\right)=d_{J}\left(r^{n+1}-(r-1)^{n+1}\right)\left(e_{1} \cdots e_{n+1}\right) .
$$

We have $c\left(K_{M}^{r} \otimes K_{M}^{-1}\right)=(r-1) c\left(K_{M}\right)=-(r-1) c_{1}(M)=(r-1) c(N)$ by (3.6), where $N$ is the normal bundle of $M$ with respect to the immersion $J$ of $M$ into $A$. Let $S$ be the singularities of $X=J(M)$ and, as in the proof of Lemma 3, let $U_{\varepsilon}(\varepsilon>0)$ be a neighborhood of $J^{-1}(S)$ such that the measure of $U_{\varepsilon}$ tends to zero as $\varepsilon$ tends to zero. For each $\varepsilon, c\left(K_{M}^{r} \otimes K_{M}^{-1}\right)$ is represented by $(r-1) J^{*} h_{J}+\eta_{\varepsilon}$, where $\eta_{\varepsilon}=0$ outside $U_{\varepsilon}$. If $r>1$, then $(r-1) J^{*} h_{J}$ is positive definite everywhere, and from this we conclude as indicated in the proof of Lemma 3 that $H^{q}\left(M, K_{M}^{r}\right)=0$ for $q>0$ provided $r>1$. Therefore we get also

$$
\operatorname{dim} H^{0}\left(M, K_{M}^{r}\right)=d_{J}\left(r^{n+1}-(r-1)^{n+1}\right)\left(e_{1} \cdots e_{n+1}\right) \quad \text { for } r>1 .
$$

Remark. It can be proved that the Ricci tensor $R_{k j}$ of the Kähler metric on $M$, which is the pullback of a flat Kähler metric in $A$, is negative everywhere, and is negative definite almost everywhere if $E(M) \neq 0$. Since $c\left(K_{M}\right)$ is represented by the $(1,1)$-form $-\frac{1}{2}(i / \pi) R_{k j} d z^{k} \wedge d \bar{z}^{j}, c\left(K_{M}\right)$ is positive definite almost everywhere. From this fact we can also prove the vanishing of cohomology groups discussed above and also in the proof of Lemma 3. 


\section{Bibliography}

[1] F. Hirzebruch, Topological methods in algebraic geometry, Springer, New York, 1966.

[2] S. Kobayashi, Transformation groups in differential geometry, Springer, Berlin, 1972.

[3] K. Kodaira, On a differential geometric method in the theory of analytic stacks, Proc. Nat. Acad. Sci. U.S.A. 39 (1953) 1268-1273.

[4] K. Kodaira \& D. C. Spencer, [a]: Divisor class groups on algebraic varieties, Proc. Nat. Acad. Sci. U.S.A. 39 (1953) 872-877. [b]: On a theorem of Lefschetz and the lemma of Enriques-Severi-Zariski, Proc. Nat. Acad. Sci. U.S.A. 39 (1953) $1273-1278$.

[ 5 ] P. Lelong, Intégration sur un ensemble analytique complexe, Bull. Soc. Math. France 85 (1957) 239-262.

[6] Y. Matsushima \& W. Stoll, Ample vector bundles on compact complex spaces, to appear.

[ 7 ] T. Nagano \& B. Smyth, Minimal varieties in tori, to appear.

[ 8 ] S. Sternberg, Lectures on differential geometry, Prentice-Hall, Englewood Cliffs, 1965.

[9] A. Weil, Introduction à l'étude des variétés kählériennes, Hermann, Paris, 1958.

UNIVERSITY OF Notre DAME 